\title{
Cultural Expressions of Bodily Awareness Among Chronically Ill Filipino Americans
}

\section{Gay Becker, PhD}

Institute for Health and Aging,

University of California, San Francisco, Calif

\begin{abstract}
PURPOSE To describe Filipino Americans' cultural traditions surrounding bodily awareness, especially how the principle of balance informs their views, and the link to self-management of chronic illness.

METHODS This qualitative study used semistructured interviews with 85 Filipino Americans between the ages of 46 and 97 years. Volunteers were recruited from numerous health care sites in 1 geographic location in the United States. Respondents had 1 or more chronic illnesses. Taped and transcribed interviews were coded and evaluated for themes.
\end{abstract}

RESULTS The concept of balance was central to Filipino Americans' portrayal of bodily awareness of signs and symptoms related to chronic illnesses, as well as to actions they took to manage their chronic illnesses. Efforts were made to control chronic illnesses through a variety of self-care practices. Diet posed a particular challenge because of the symbolic importance of food in Filipino culture and its use in the maintenance of social relationships.

CONCLUSIONS The ways in which Filipino Americans combine attention to the body, values of balance and harmony, and emphasis on social well-being result in heightened attention to bodily processes. Filipino Americans' emphasis on bodily awareness suggests that this particular cultural strength can be used to enhance chronic illness management. Awareness of the cultural traditions of Filipino Americans can facilitate patient education about how to manage chronic illnesses.

Ann Fam Med 2003;1:113-118. DOI: 10.1370/afm. 39.

\section{INTRODUCTION}

S elf-monitoring is viewed as a necessary part of effective management for chronic illnesses, ${ }^{1,2}$ but such skills are not automatic. Learning to manage a chronic illness effectively can take years and can be seen as a lifelong process. ${ }^{3,4}$ Although the literature on self-management infrequently attends to cultural factors, ${ }^{5-7}$ when it does address such concerns, culture-specific ingredients that affect how people manage their illnesses have been shown to be important. ${ }^{8-10}$ In particular, a focus on bodily awareness - its cultural explicitness and its importance for action in everyday life - has shown how bodily experiences reflect culture. ${ }^{3,11-15}$

The portrayal of bodily experience - the connection between how people experience their bodies and how they talk about them - offers important clues for illness management, especially for self-monitoring and selfcare techniques. In this article, I explore the relationship between cultural expressions of bodily knowledge and the development of illness management skills by examining the statements of Filipino Americans who had 1 or more chronic illnesses and who were engaged in efforts to maintain control of illnesses, such as diabetes, hypertension, and asthma. The pur- 
pose of this study was to describe Filipino Americans' cultural traditions surrounding bodily awareness, especially how the principle of balance informs their views, and the link to self-management of chronic illness. Awareness of cultural traditions among immigrant populations such as Filipino Americans might be important to clinicians attempting to improve the quality of care for chronic diseases for this population.

\section{The Social Context of Health Among Filipino Americans}

The principle of balance, or timbang, is central to Filipino concepts of health, and balance is sought in all social relationships. Researchers studying different parts of the Philippines have recorded a range of cultural practices around health, ${ }_{1}^{16-20}$ but a dominant, unifying theme is that of balance. Anderson ${ }^{16}$ suggests that health is thought to be a result of balance; illness is usually the result of some imbalance. Health and illness are closely linked with sociality: the concepts used by Filipinos to understand the nature, cause, and treatment of illness are consistent with and informed by those that order their social lives. ${ }^{16}$ Rosaldo $^{20}$ found that health, happiness, and sociality were all intimately linked.

Humoral pathology concepts are pervasive in the Philippines; they reflect a complicated system based on a few fundamental principles, one of which is the range of hot-cold beliefs concerning principally humoral balances in the body and food and dietary balances. ${ }^{16}$ According to this theory, rapid shifts, especially from "hot" to "cold," cause illness and disorders. Optimal health is maintained in part by keeping the body in a "warm" condition. These principles are salient for Filipino Americans' cultural understandings of their bodily experience in the United States.

\section{METHODS}

The findings are based on 2 large qualitative studies that examined the same questions about how people live with and manage their chronic illnesses. The first study was a 10-year anthropological study of the experiences of ethnic minority elders with chronic illness in which illnesses were not specified. The second was a 4 -year study that asked the same questions but was specific to asthma. The studies are combined here to illustrate the pervasiveness of certain themes for older Filipino Americans. The most common illnesses were diabetes mellitus, asthma, and heart disease or hypertension. The total sample of Filipino Americans was 85; respondents were between the ages of 46 and 97 years. In addition, 3 other ethnic groups were studied using the same approach: Latinos, African Americans, and Cambodian Americans. Each ethnic group was analyzed separately.
They are omitted from this analysis. This substudy focuses only on cultural patterns specific to Filipino Americans, enabling a more in-depth analysis.

Filipino Americans do not represent a homogeneous group. As Anderson ${ }^{16}$ points out, Filipinos in the Philippines reflect considerable diversity, which is intensified in the United States. Filipinos do share underlying values about the importance of bodily awareness, however, which became readily apparent in this analysis. Filipino Americans in this study can be roughly divided into 2 groups: recent US immigrants who do not necessarily intend to stay in the United States, and those who immigrated more than 10 years ago and have no plans to return to the Philippines.

Respondents were recruited from more than 20 sources, including community health clinics, senior centers, acute care hospitals, and home care services, between December 1994 and September 2001. The sample reflected a range of illness severity from mild to severe. The study protocol and consent form were approved by the Institutional Review Board, Committee on Human Research, University of California, San Francisco.

According to key tenets of the in-depth interviewing approach, ${ }^{21-23}$ respondents were interviewed 3 times in a 1-year period. Semistructured hour-long interviews addressed interpretations of illness, self-care practices, and use of and access to health care. Interviews were tape-recorded and transcribed verbatim. Respondents were interviewed in their language of choice, either Tagalog or English. With the exception of 2 respondents who were interviewed in English, respondents were interviewed in Tagalog by a fluent Tagalog speaker.

Qualitative content analysis was used to analyze the data ${ }^{24,25}$ according to a specific procedure: core categories that repeatedly reappeared in the data were classified, and selected transcripts were read by the entire team to generate consensus in coding categories, which led to the development of more than 100 codes. Codes included body, self-care, causation, and illness knowledge. It was through this coding process that I was first alerted to Filipino Americans' particular emphasis on the body, which was also reflected in a case-by-case narrative analysis, ${ }^{3}$ in which emphasis was given to how respondents expressed bodily comfort and distress and the actions they took to feel better.

\section{RESULTS}

\section{Demographics}

The 85 respondents ranged in age from 46 to 97 years, with a mean age of 69 years; $52 \%$ were women and 
$48 \%$ were men. Ninety-five percent spoke Tagalog, and $95 \%$ also spoke English. Because of the long-term colonial relationship of the United States to the Philippines, Filipinos were familiar with Western culture before they came to the United States. Respondents reflected diversity in socioeconomic status, ranging from middle-income home owners who had medical insurance to those who were poor, lived in lowcost housing, and received either Medicaid or were uninsured. All respondents were living in the community at the time of the study. Recent immigrants lived in single-room-only dwellings and small apartments, whereas those who immigrated more than 10 years ago lived in suburban areas. Despite these differences, the importance of bodily experience was a pervasive theme. Table 1 displays the demographic characteristics of the study population.

\section{Body Imbalances}

Respondents who were recent immigrants to the United States described 2 types of body imbalances as explanations for why they became ill: (1) those caused by humoral pathology and (2) those caused by stress. Moving from a hot place, the Philippines, to a cold place, a coastal US city, was repeatedly given as a reason for the onset of illness. For example, a 65-year-old woman, when asked why she thought she had developed a heart problem in the United States, said, "The climate is different. It's because we don't perspire. That's what they say. It's so cold your body doesn't sweat. That's why things like cholesterol aren't being sweated out."

Imbalance in the form of worry and overwork is also seen as creating stress and causing illness. ${ }^{16} \mathrm{~A}$ typical comment was made by a 63-year-old woman who said, "In the Philippines I already had high blood because I worry too much. I didn't get to sleep much. Of course I had to work hard because I was the head person. That's probably why my blood pressure went up. That's what caused me to be sick."

Many respondents who immigrated in later life had a chronic illness diagnosed for the first time in the United States. Most reported infrequent physician visits before immigration because of the expense, but once in the United States, $87 \%$ had health insurance. A 60-year-old woman explained, "I only got sick here. Imagine if you have 9 children and you are caring for all of them. And so that stress built up on my body. And that's why I think all of the sickness came out of my body."

\section{Bodily Awareness}

New diagnoses heightened respondents' attention to their bodies. The diagnosis of chronic illness is often
Table 1. Demographic Characteristics

\begin{tabular}{|c|c|c|}
\hline Characteristics & $\begin{array}{l}\text { Number } \\
(\mathrm{N}=85)\end{array}$ & $\begin{array}{l}\text { Percent } \\
(100 \%)\end{array}$ \\
\hline \multicolumn{3}{|l|}{ Age } \\
\hline Age range, $y$ & $46-97$ & \\
\hline Mean age, y & 69.09 & \\
\hline \multicolumn{3}{|l|}{ Sex } \\
\hline Women & 44 & 51.8 \\
\hline Men & 41 & 48.2 \\
\hline \multicolumn{3}{|l|}{ Marital status } \\
\hline Married & 66 & 78.6 \\
\hline $\begin{array}{l}\text { Unmarried (widowed, divorced, } \\
\text { separated, never married) }\end{array}$ & 18 & 21.4 \\
\hline \multicolumn{3}{|l|}{ Education } \\
\hline No education & 1 & 1.2 \\
\hline Grade school & 40 & 47.1 \\
\hline Some high school & 13 & 15.3 \\
\hline High school graduate & 14 & 16.5 \\
\hline Some college or vocational training & 3 & 3.5 \\
\hline College graduate & 11 & 12.9 \\
\hline Postgraduate & 2 & 2.4 \\
\hline Other & 1 & 1.2 \\
\hline \multicolumn{3}{|l|}{ Work life } \\
\hline Retired & 64 & 75.3 \\
\hline Currently working & 5 & 5.9 \\
\hline Homemaker & 13 & 15.3 \\
\hline Unemployed & 1 & 1.2 \\
\hline Disabled & 2 & 2.4 \\
\hline \multicolumn{3}{|l|}{ Occupation } \\
\hline $\begin{array}{l}\text { Business manager, administrative, } \\
\text { minor professional }\end{array}$ & 8 & 9.4 \\
\hline Technical, semiprofessional & 2 & 2.4 \\
\hline Clerical, sales & 2 & 2.4 \\
\hline Skilled labor & 22 & 25.9 \\
\hline Machine operation, semiskilled & 25 & 29.4 \\
\hline Unskilled labor & 10 & 11.8 \\
\hline Farm labor, menial & 3 & 3.5 \\
\hline Homemaker & 13 & 15.3 \\
\hline \multicolumn{3}{|l|}{ Religious affiliation } \\
\hline Roman Catholic & 75 & 89.3 \\
\hline Protestant, other* & 7 & 8.4 \\
\hline No religious affiliation & 2 & 2.4 \\
\hline
\end{tabular}

accompanied by increased attention to bodily processes in the general US population. ${ }^{3}$ Respondents expressed close attention to bodily processes. In addition to discussing bodily routines, they exhibited a particular tendency to analyze any signs, small or large, that their bodies were not functioning optimally, a tendency common to the general population with chronic illnesses that are accompanied by symptoms, whether sporadic or relentless. ${ }^{3}$ This phenomenon was pronounced among Filipino American respondents. They not only experienced bodily signs, they articulated those signs in detail and pondered their meaning. They 
were preoccupied with signs or symptoms that they considered warnings of their illnesses. The signs that respondents described were not necessarily widely held symptoms of a particular health condition but could be highly idiosyncratic. For example, one 75-year-old man said, "When my high blood pressure acts up, it's like my body stiffens. I feel like my tongue swells up, and then I have to take my medicine right away. Last night I was like that. I guess it's because my pressure went up again."

Restraint in emotions was also described in striving to regain balance. A 70-year-old man described a 2-part process: he addressed issues of regaining balance first, and then described a period of analysis: "I just calm myself. I don't pay attention to my sickness. I don't feed it with concern. I just relax, and then it goes away. You just have to ignore it. Afterwards you have to think about what kind of medicine you will take. Why did it happen? Of course, you can sort of think about what will get rid of it."

\section{Controlling Illness}

Respondents did not link bodily monitoring simply to their chronic illnesses but to their roles in the family, to the importance of membership in the social group, and the desire to participate fully in family and group life. Filipino Americans expressed a sense of responsibility for maintaining good health, but their responsibility was to the family and social group rather than individual responsibility as an end in itself. Because of their sense of responsibility, they took the initiative in seeking medical care and following medical regimens.

One of the main efforts respondents made to control their illnesses was through food. Food is very important in Filipino culture and symbolizes sharing and reciprocity. ${ }^{26-28}$ Relationships are reestablished through food. Food is always served to guests; to not eat the host's food is to reject these social ties, or comparadizo. To refuse food, even for health reasons, has implications for the quality of the food served and brings shame on the person offering it. Managing their illnesses by controlling what they ate thus created conflict for many respondents who had diabetes and high blood pressure.

Women and men struggled with the limitations they experienced around food. For example, a 70-yearold man made a typical comment about trying to deal with dietary constraints: "When I eat the things that the doctor told me I shouldn't, then for sure I feel the effects. I have a hard head. But I don't, anymore. I am scared now because it might get really bad and then the doctor will have a hard time treating me."

Respondents recited their usual diets, especially favorite foods they relied on for satisfying meals, as well as those they gave up or ate in greater moderation. Reducing rice intake was especially difficult. For example, a 62-year-old woman who had high blood pressure said: "Just one bowl of rice, because I am taking care of my high blood. Because sometimes I can't see when I get up too quickly." Signs, such as not being able to see, were reminders to be careful that respondents, such as this woman, did not ignore. Instead, they heeded what they considered to be warnings and renewed their efforts to control the illness.

Watching one's diet was viewed as an important component of healthy living. Respondents who did not have illnesses that particularly warranted care with diet were nevertheless careful about their diet. A 70year-old man reflected the high health consciousness of others in the sample when he said, "I control what I eat because it's better for me. And I think a thin body is better. When you are overweight it's hard. You get high blood, heart attacks."

\section{Self-Care}

Good health was highly valued and associated with the ability to present a public picture of well-being and youthfulness. A healthy, well-kept appearance was central to the respondents' lifestyle regardless of their income level. Good health was considered something to be fostered to participate fully in the life of the community for one's own sake, as well as for one's responsibility to the social group.

The principle of balance informed and enhanced a basic approach to self-care. These 2 philosophies dovetailed to affect the respondents' outlook, as one man commented, "I don't eat anything that is not good for me, and I also exercise now. Every morning when I wake up so that I will feel better. Before my head used to hurt because I would eat too much rice. It's like my feelings are better now. I just feel better now." This man's comments speak not only to moderation and balance, but to how staying healthy and fit is integrated with emotion. Rosaldo's Filipino respondents were interested in feelings because affective life had consequences for health, cooperation, daily labor, and political debate. ${ }^{20}$ Similarly, Filipino Americans in this research volunteered their feelings because feelings were integrated into their overall view of health and life.

Awareness of the importance of self-care, especially the health benefits of exercise and careful diet, was commonplace among these respondents. Exercise such as walking was valued as a means of preserving or promoting health. Comments typical of these interviews included: "I walk everyday. I take my time. I don't hurry. As long as I am walking a good distance," and "We walk everywhere. I joined all types of things, like the exercise class here." 


\section{DISCUSSION}

Although there is a growing body of literature on Filipinos immigrating to the United States ${ }_{1}^{29-32}$ the work done to date on Filipino Americans' health beliefs and practices has been extremely limited. With only a few exceptions, little research has examined bodily awareness of illness, the application of principles of good health, or the management of illness. ${ }^{10,26,28,33}$ Among Filipino Americans in this study, bodily awareness was a part of their daily routines as well as a built-in part of their social lives. Bodily awareness facilitated efforts to maintain the balance viewed as so important to health and well-being. In a study of Filipino Americans' attitudes about tuberculosis, respondents cited the importance of correcting bodily imbalances. ${ }^{33}$ In the current study, paying attention to signs and symptoms was not only the centerpiece of a carefully articulated body consciousness, it reflected an overall social emphasis on harmony and balance in which efforts to control illness and to initiate and maintain self-care practices were affected. Feelings, or emotions, were a central aspect of bodily awareness, reflecting, overall, a holistic approach to the body that contrasts with American mainstream and biomedical tendencies to separate mind and body. ${ }^{3}$

Discussion of bodily processes and interventions to improve health was part of the social fabric of everyday life. The root metaphor of balance in health and religion is expressed in the key value of reciprocity in social interaction. ${ }^{16}$ Peers and family members played an important role in elevating body consciousness and making it a key social value. The articulation of bodily awareness through continuous social interchange strengthened values of self-care and reinforced efforts to monitor illnesses and control them. Body consciousness was thus a social norm. At the same time, however, cultural values expressed through the medium of food posed a dilemma for most respondents in this research. In another study, rice meals were viewed by some as proper, complete, and healthy, and were associated with Filipino food pathways, but also posed a quandary because of the emphasis on high-fat content and carbohydrates. ${ }^{27(\mathrm{p} 187)}$ Respondents in this study faced the same health and cultural dilemmas associated with food.

Despite the emphasis on humoral concepts of health in the Philippines, Western biomedicine is an important influence on Filipino Americans' lives in the United States. Anderson ${ }^{16}$ notes that the basic logic of health and illness involves both prevention (avoidance of inappropriate behavior that causes imbalance) and curing (by restoring balance), and that paralleling a comprehensive belief system is the understanding that certain diseases and disorders are best treated by modern clinical medicine because they operate by a differ- ent logic. He notes that little conflict is perceived between these 2 systems of explanation by Filipinos. Their views about health and the management of illness, while grounded in different cultural traditions, are complementary to mainstream American cultural views of health and illness that illness can and should be controlled and that the individual is responsible for doing so. The biomedical model is widespread in the Philippines, and both persons who immigrated long ago as well as recent immigrants are familiar with it. They subscribe to biomedical notions about illness management, prevention, and self-care, as well as to the value of health practices that foster good health overall. Recent immigrants make the most of the heightened access to care that they find in the United States. ${ }^{30}$

Physicians who treat Filipino American patients should be aware of cultural attitudes held in common that affect how patients view their bodies and their health. The importance of bodily awareness among Filipino Americans offers a particular opportunity for working together with patients to develop effective illness management plans. It should be recognized, however, that managing dietary needs might pose a particular challenge for Filipino Americans of all ages because of the cultural considerations with which they are perennially faced in social situations.

This research has implications for clinicians who treat chronic disease in other immigrant populations. Awareness of how cultural values affect views about health and illness in a given group might improve quality of care for chronic disease. Such awareness can provide insights into how best to help patients understand their illnesses and facilitate the incorporation of illness management plans into their lives.

This study had several limitations. The sample size was small and was drawn from a single geographic location. Additionally, the sample was drawn from volunteers in their forties and older who were recruited through a variety of means, such as flyers and referrals. Furthermore, the interviewer translated and transcribed interviews she had conducted because of a dearth of trained transcriptionists who were adequately bilingual. Hence, these findings might not apply to all Filipino Americans. Although the cultural traditions described in this article might have greater applicability to older than younger patients, the high proportion of multigenerational Filipino American families who maintain close ties through co-residence and close connections with the homeland suggests that these cultural traditions might be pervasive among the younger generation as well. It should be kept in mind that the goal of qualitative research is to uncover and delineate new perspectives for research and practice rather than to make generalizable statements. 


\section{CONCLUSIONS}

The ways in which Filipino Americans combine attention to the body, values of balance and harmony, and emphasis on social well-being result in heightened attention to bodily processes and to the culturally elaborated care of the body. Filipino Americans' emphasis on bodily awareness suggests that this particular cultural strength can be used to enhance chronic illness management. Awareness of the cultural traditions of Filipino Americans can be used to facilitate patient education about how to manage chronic illnesses.

To read commentaries or to post a response to this article, see the online version at http://annfammed/cgi/content/full/1/2/113.

Key words: Asian Americans, Cultural Characteristics, Chronic Disease, Self Care, Life Style

Submitted November 26, 2002; accepted April 8, 2003.

\section{Financial support}

This work is based on research from 2 projects funded by the National Institute on Aging, National Institutes of Health for which Gay Becker was principal investigator: R37 AG11144, "Cultural Responses to Illness in the Minority Aged," and RO1 AG16608, "Age and Illness Management in Later Life."

\section{Acknowledgments}

Many thanks to Edwina Newsom, Leilani Canalita, and Kumiko Shimizu for their work on these projects.

\section{References}

1. Ferzacca S. "Actually, I don't feel that bad": managing diabetes and the clinical encounter. Med Anthropol Q 2000;14:28-50.

2. Strauss A, Corbin J, Fagerhaugh S, Glaser BG, Maines D, Scuzek B, Wiener CL. Chronic Illness and the Quality of Life. St Louis, Mo: CV Mosby Co; 1984.

3. Becker G. Disrupted lives: How people create meaning in a chaotic world. Berkeley, Calif: University of California Press; 1997.

4. Charmaz K. Good days, bad days: the self in chronic illness and time. New Brunswick, NJ: Rutgers University Press; 1991.

5. Katz PP. Education and self-care activities among persons with rheumatoid arthritis. Soc Sci Med 1998;46:1057-1066.

6. Clark NM, Becker MH, Janz NK, Lorig K, Rakowski W, Anderson L. Self-management of chronic disease by older adults: a review and questions for research. J Aging Health 1991;3:3-27.

7. Norborn, JEK, Bernard SL, Konrad TR, et al. Self-care and assistance from others in coping with functional status limitations among a national sample of older adults. J Gerontol B Psychol Sci Soc Sci. 1995;50:S101-109.

8. Silverman M, Musa D, Kirsch B, Siminoff LA. Self-care for chronic illness: Older African Americans and whites. J Cross-Cult Gerontol 1999; 14:169-189.

9. Garro L. Explaining high blood pressure: Variation in knowledge about illness. Am Ethnol 1988;15:98-119.
10. Becker G, Beyene Y, Newsom EM, Rodgers DV. Knowledge and care of chronic illness in three ethnic minority groups. Fam Med 1998;30:173-178.

11. Becker A. Body, self, and society: the view from Fiji. Philadelphia, Pa: University of Pennsylvania Press; 1995.

12. Jackson M. Things as they are: new directions in phenomenological anthropology. Bloomington, Ind: Indiana University Press; 1996.

13. McCallum C. The body that knows: from Cashinahua epistemology to a medical anthropology of lowland South America. Med Anthropol Q 1996;10:347-372.

14. Roseman M. Healing sounds from the Malaysian rainforest: Temiar music and medicine. Berkeley, Calif: University of California Press; 1991.

15. Merleau-Ponty M. Phenomenology of perception. London: Routledge; 1962.

16. Anderson J. Health and illness in Pilipino immigrants. West J Med 1983;139:811-819.

17. Nichter M, Nichter M. Acute respiratory illness: Popular health culture and mother's knowledge in the Philippines. Med Anthropol 1994;15:353-375.

18. Edman JL, Kameoka VA. Cultural differences in illness schemas: An analysis of Filipino and American illness attributions. J Cross-Cult Psychol 1997;28:252-266.

19. Lieban RW. Sex differences and cultural dimensions of medical phenomena in a Philippine setting. In: Morley P, Wallis R, eds. Culture and Curing: Anthropological Perspectives on Traditional Medical Beliefs and Practices. Pittsburgh, Pa: University of Pittsburgh Press; 1979:99114.

20. Rosaldo M. Knowledge and passion: llongot notions of self and social life. Cambridge, UK: Cambridge University Press; 1980.

21. Spradley JP. The ethnographic interview. New York, NY: Holt, Rinehart, \& Winston; 1979

22. Rubinstein R. Stories told: in-depth interviewing and the structure of its insights. In: Reinharz S, Rowles GD, eds. Qualitative Gerontology. New York, NY: Springer; 1987:128-146.

23. Langness LL, Frank G. Lives: an anthropological approach to biography. Novato, Calif: Chandler and Sharp; 1981.

24. Hammersley M, Atkinson P. Ethnographic principles in practice. London: Tavistock; 1986.

25. Clark MM, Anderson B. Culture and aging. Springfield, III: Charles C. Thomas; 1967.

26. Farrales LL, Chapman GE. Pilipino women living in Canada: constructing meanings of body, food, and health. Health Care Women Int 1999;20:179-194.

27. Law L. Home cooking: Filipino women and geographies of the senses in Hong Kong. Ecumene 2001;8:264-283.

28. DiPasquale-Davis J, Hopkins SJ. Health behavior of an elderly Filipino group. Public Health Nurs 1997;14:118-122.

29. Becker G. Dying away from home: quandaries of migration for elders in two ethnic groups. J Gerontology B Psychol Sci Soc Sci 2002:57:S79-S95.

30. Becker G, Beyene Y, Canalita LC. Immigrating for status in late life: effects of globalization on Filipino American veterans. J Aging Studies 2000; 14:273-291

31. Bonus R. Locating Filipino Americans: Ethnicity and the Cultural Politics of Space. Philadelphia, Pa: Temple University Press, 2000.

32. Espiritu YL. Filipino American Lives. Philadelphia, Pa: Temple University Press, 1995.

33. Yamada S, Caballero J, Matsunaga DS, Agustin G, Magana M. Attitudes regarding tuberculosis in immigrants from the Philippines to the United States. Fam Med 1999;31:477-482. 